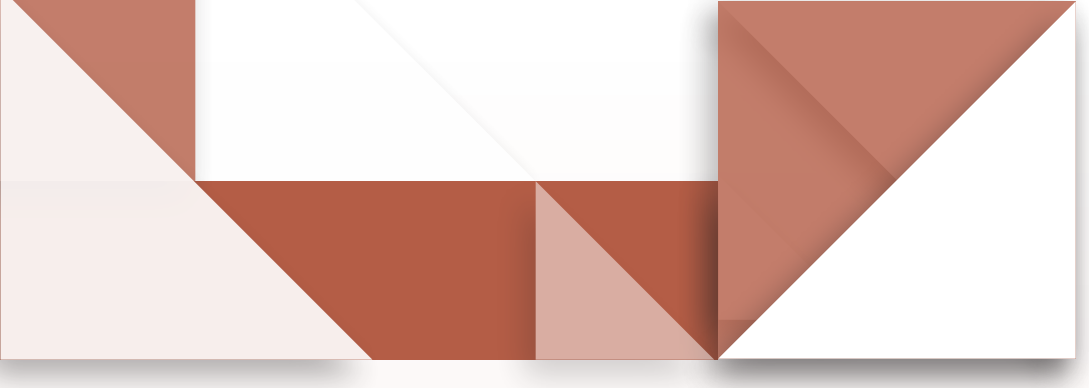

\section{Contribuciones de la} actividad experimental al pensamiento científico de estudiantes con diversidad funcional

- Contributions of Experimental Activity to the Scientific Thinking of Students with Functional Diversity

- Contribuições da atividade experimental para o pensamento científico de alunos com diversidade funcional

\section{Resumen}

En este artículo se reportan, en forma de narrativa, los resultados del estudio de caso de un estudiante (Carlos) diagnosticado con trastorno de déficit de atención, hiperactividad e impulsividad y trastorno mixto de habilidades escolares, quien presentaba dificultad en la comprensión de los conceptos fundamentales de la ciencia, apatía por la escuela, y un cuadro de aislamiento debido a la falta de reconocimiento por parte de sus maestros y compañeros de estudio. Estas problemáticas llevaron al planteamiento de la pregunta orientadora del caso: ¿̇cómo promover habilidades de pensamiento científico en estudiantes con diversidad funcional? Esto llevó al propósito central de la intervención: proponer la actividad experimental exploratoria en torno al fenómeno de flotabilidad como una aliada en el aula diversa funcional. La propuesta se fundamentó en torno a construcciones teóricas sobre la diversidad funcional, la actividad experimental, las habilidades de pensamiento científico y el fenómeno de flotación. La estrategia se desarrolló como un sistema en espiral: inició con un estudio diagnóstico sobre las dificultades, los gustos, los intereses y las necesidades del estudiante y llegó hasta las evidencias del desarrollo de habilidades de pensamiento científico, habilidades que permitieron fortalecer el autorreconocimiento, estimular el acercamiento a pensar la actividad científica y promover el sentido creativo del estudiante. Todo esto se logró mediante un seguimiento constante del proceso de enseñanza y la receptividad por parte del estudiante. Se pudo valorar los progresos sobre sus niveles de atención, fortalecimiento de habilidades sociocientíficas y aprendizajes significativos.

Palabras clave:

diversidad funcional; actividad experimental exploratoria; habilidades de pensamiento científico; enseñanza de las ciencias

\section{Edwin García Arteaga* Eliana Bolaños Ortega ${ }^{\star *}$}




\section{Abstract}

This article presents, in the form of a narrative, the results of the case study of a student (Carlos) diagnosed with Attention Deficit Disorder, Hyperactivity and Impulsivity and Mixed School Skills Disorder, who presented difficulty in understanding the fundamentals concepts of science, apathy for school, and a picture of isolation due to lack of recognition from their teachers and fellow students. These problems led to posing the guiding question of the case: how to promote scientific thinking skills in students with functional diversity? This led to the central purpose of the intervention: to propose exploratory experimental activity around the phenomenon of buoyancy as an ally in the diverse functional classroom. The proposal was based on theoretical constructions on functional diversity, experimental activity, scientific thinking skills and the floating phenomenon. The strategy was developed as a spiral system: it began with a diagnostic study of the difficulties, tastes, interests and needs of the student and reached the evidence of the development of scientific thinking skills, skills that made it possible to strengthen self-recognition, stimulate the approach to thinking about scientific activity and promoting the creative sense of the student. All this was achieved through constant monitoring of the teaching process and the receptivity on the part of the student. It was possible to assess the progress on their levels of attention, strengthening of socio-scientific skills and significant learning.

Keywords:

functional diversity: exploratory experimental activity; scientific thinking skills; science teaching

\section{Resumo}

Neste artigo relatam-se, em forma de narrativa, os resultados do estudo de caso de um aluno (Carlos) com diagnóstico de Déficit de Atenção, Hiperatividade e Impulsividade, assim como, Transtorno de Habilidades Escolares Mistas, quem apresentava dificuldade na compreensão dos conceitos fundamentais da ciência, apatia pela escola, um quadro de isolamento graças à falta de reconhecimento de seus professores e colegas de estudo. Esses problemas levaram ao levantamento da questão norteadora do caso: Como promover habilidades de pensamento científico em alunos com diversidade funcional? Isso levou ao propósito central da intervenção:propor a atividade experimental exploratória em torno do fenômeno da flutuabilidade como um aliado na sala de aula funcional diversa. A proposta foi baseada em torno de construções teóricas sobre diversidade funcional, atividade experimental, habilidades de pensamento científico e o fenômeno flutuante. A estratégia desenvolveu-se como um sistema em espiral: partiu comum estudo diagnóstico sobre as dificuldades, gostos, interesses e necessidades do aluno até as evidências do desenvolvimento de habilidades de pensamento científico, habilidades que permitiram fortalecer o auto-reconhecimento, estimular a abordagem para pensar a atividade científica e promover o sentido criativo do estudante. Tudo isto conseguiu-se através do acompanhamento constante do processo de ensino do professor e da receptividade por parte do aluno. Foi possível avaliar a evolução dos seus níveis de atenção, fortalecimento de habilidades sociocientíficas e aprendizagens significativas.

Palavras-chave:

diversidade funcional: atividade experimental exploratória; habilidades de pensamento científico; ensino de ciências 


\section{Introducción}

Investigaciones sobre la diversidad en la enseñanza de las ciencias señalan tres aspectos relevantes para el planteamiento del presente trabajo. El primero hace visible una realidad del aula: allí se encuentran diferentes tipos de aprendices, que poseen rasgos únicos con los que adquieren capacidades genuinas para aprender en medio de una gran diversidad funcional (DF) (Harlem, 1998; McGinnis y Kahn, 2014). El segundo reconoce que la labor de los maestros es hacer una lectura apropiada de esta diversidad y buscar estrategias que le permitan acercar el mundo científico a todos sus aprendices (García, 2018; Kahn, 2018). Un tercer aspecto hace referencia a estudios que revelan las concepciones segregadoras de los maestros sobre el trabajo con estudiantes que presentan diversidad funcional, puesto que deben asumir esta realidad sin las herramientas necesarias. Esto como una consecuencia de la falta de formación de los maestros de ciencias naturales, lo cual les impide adoptar estrategias adecuadas para las capacidades de cada estudiante desde etapas iniciales, específicamente de aquellos que tienen diagnósticos enmarcados en las necesidades educativas especiales y cuyo desempeño difiere del promedio de la clase (López et al., 2012; Mastropieri y Scruggs, 2017).

La conjunción de estos aspectos enmarca la problemática de este caso en la falta de capacitación de los maestros de ciencias naturales para atender la diversidad funcional en el aula. Esto se evidencia cuando el docente emplea continuamente medios impresos y expositivos (guías, talleres y clases magistrales) como única estrategia para la enseñanza. El problema es que en estas actividades se exige de los estudiantes con diversidad funcional el uso habilidades del lenguaje y de vocabulario técnico-científico, así como altas demandas de memoria y atención, aspectos en los que precisamente se encuentran las necesidades de apoyo de la mayoría de los diagnósticos de esta población (Mastropieri, 2006).

Usualmente, la actividad experimental en la enseñanza de las ciencias es utilizada para demostrar o comprobar una ley o una teoría, y el estudiante se ve obligado a cumplir con una guía o receta de pasos para alcanzar el resultado final de la demostración, dejando por fuera los procesos de desarrollo de pensamiento al diseñar, manipular, interpretar y explicar fenómenos, procesos que han sido identificados históricamente en el desarrollo de una ciencia. La actividad experimental exploratoria está más en función de los procesos del pensamiento científico (tensiones, valores y creencias del experimentador) que de los productos de la ciencia (principios y leyes). Es asumida como una actividad que tiene una riqueza conceptual, ya que le permite al maestro de ciencias reflexionar sobre la imagen de ciencia que tiene, los conceptos y las relaciones que existen entre estos y la realidad que se pretende interpretar en la búsqueda de nuevas significaciones, propias de los problemas particulares de su entorno.

El aporte más significativo en este campo corresponde a Andy Pickering (1989), quien establece que en la actividad experimental entran en juego tres elementos:

- Un procedimiento material: disponer de los aparatos e instrumentos necesarios, verificar y controlar su funcionamiento implica un conocimiento práctico.

- Un modelo instrumental: en el diseño, realización e interpretación del experimento, es fundamental la comprensión conceptual del funcionamiento de aparatos e instrumentos. 
El experimentador tiene un modelo conceptual que le permite identificar, aplicar y usar los aparatos.

- Un modelo fenoménico: se utiliza en la comprensión conceptual de los aspectos del mundo fenomenológico que está siendo estudiado por parte del experimentador. Sin él, los resultados carecerán de sentido y significación, y no podrían ser interpretados (García, 2015).

La actividad experimental exploratoria supone observar y experimentar. Lo primero sería imposible sin la percepción de señales sensibles. La segunda es una actividad que requiere la intervención activa en los procesos naturales con el objeto de obtener respuestas a las preguntas formuladas hipotéticamente de acuerdo con un plan establecido. De otra parte, la experiencia es asumida en la interacción entre el observador y el fenómeno, enriquece sus conocimientos y resignifica los hechos (al construirlos y organizarlos). En la organización de los fenómenos naturales, la experiencia no está dada, hay que construirla. Y hacerlo es llenar de sentido una actividad en la que la práctica experimental es un medio de constante reflexión sobre el fenómeno abordado. En consecuencia, construir experiencia que sea sensible a partir de las preocupaciones individuales resulta significativo. A partir de las preguntas e indagaciones se empieza a construir conocimiento en la misma medida que surgen nuevas preguntas y por lo tanto nuevas experiencias que hacen que la imagen del fenómeno se amplíe cada vez más (García, 2011).

\section{La diversidad funcional y la actividad experimental exploratoria}

El término diversidad funcional se refiere a todas las personas que por motivos de la diferencia del funcionamiento de alguna parte de su cuerpo realizan tareas habituales de maneras no convencionales. Se utiliza en reemplazo de términos peyorativos y excluyentes como minusválido, limitado o discapacitado. Romañach y Lobato (2005), quienes proponen este término, afirman que cambiar el lenguaje ayuda a vencer la segregación y promover los procesos de normalización de la diversidad en todos los ámbitos humanos (McGinnis y Kahn, 2014). Por esto es importante que maestros y estudiantes reconozcan como algo cotidiano las diversas maneras de construir el aprendizaje que convergen en el aula de ciencias.

Por tanto, la actividad experimental exploratoria se convierte en una aliada importante de los maestros de ciencias y los estudiantes con diversidad funcional porque promueve habilidades de pensamiento científico gracias a la interacción con los aparatos e instrumentos, potencia el discurso y la argumentación, además de elevar la autoestima y la capacidad de diálogo y de ofrecer la oportunidad de hacer comprensibles los fenómenos objetos de estudio. Por esto, la actividad experimental exploratoria en la enseñanza de las ciencias se ha estructurado como pieza fundamental en la construcción del conocimiento científico de los estudiantes. Los maestros deben reconocerla desde un rol más cercano, que 
involucre las preocupaciones de los estudiantes y los sucesos cotidianos desde un contexto personal, que responda preguntas y genere nuevos interrogantes (Marín y García, 2013).

\section{La flotabilidad de los cuerpos y las} habilidades de pensamiento

La flotabilidad de los cuerpos es un fenómeno cotidiano que hace referencia al concepto fundamental de equilibrio (físico, químico, biológico y ambiental) de cuerpos que se hunden o flotan, densidad del medio, resistencia y empuje, y capacidad, volumen y forma, entre otros. De acuerdo con García et al. (2005), la enseñanza de la flotación de los cuerpos a través de la actividad experimental exploratoria puede considerar las siguientes estrategias: 1) asociar la flotación a situaciones de equilibrio (en el mar un barco flota y una aguja se hunde) y tener presente la superficie de sustentación del objeto (comparar un barco grande con uno más pequeño de la misma forma y material); 2) asociar el volumen y la forma del objeto con la resistencia del medio (un barco en agua y un barco en mercurio); 3) asociar el material del que está hecho el objeto, la forma, el tamaño y el volumen pero diferente material (objetos de cobre, hierro o aluminio sumergidos en diferentes medios agua, agua-sal, aceite), 4) explorar el comportamiento de los objetos cuando cambiamos las condiciones del medio en su temperatura o densidad (un huevo crudo en agua se va al fondo, pero al agregar sal esta condición cambia).

Conviene subrayar que se elige relacionar el fenómeno de flotación de los cuerpos en esta investigación porque responde a los gustos e intereses del estudiante protagonista del estudio de caso y por la importancia que representa vivenciar el fenómeno en clase y las oportunidades para promover las habilidades de pensamiento en estudiantes con TDAHI, quienes presentan dificultades para atender a la clase magistral o realizar actividades que impliquen lectoescritura y cálculo. Aunque las estrategias que propone García et al. (2005) no hacen referencia a estudiantes con diversidad funcional, se adapta esta secuencia de actividades experimental para llevar al estudiante con estas características a relacionar temas cotidianos, a fin de comprender los aspectos del fenómeno lejos del libro de texto.

De acuerdo con las tesis expuestas anteriormente por Pickering (1986) y organizadas por García (2011), es importante anotar que la actividad experimental exploratoria puede ser considerada como una estrategia fundamental para promover habilidades de pensamiento científico en personas con diversidad funcional. El reconocimiento de los aparatos e instrumentos que hacen parte del fenómeno es importante en una etapa inicial; los niños pueden y deben manipular los aparatos, jugar con ellos, reconocer sus partes, hacer registros y medidas, por ejemplo, identificar una balanza, medir con un dinamómetro y reconocer su funcionamiento, ya que esto les brinda seguridad al momento de experimentar. Además, promover el diseño de aparatos funcionales y acordes a la edad mental del niño provoca la creatividad y el desarrollo de pensamiento.

Todas estas actividades experimentales exploratorias ayudarán a comprender los fenómenos estudiados de acuerdo con el nivel de escolaridad y pensamiento del niño, porque él puede comprender conceptos asociados desde el nivel macroscópico y posteriormente relacionar variables. Por ejemplo, para el caso de esta investigación, fue necesario llevar al estudiante a identificar las características de los cuerpos que flotan, los que se hunden y las condiciones en que un objeto puede flotar o sumergirse. Toda esta trama tejida entre actividad experimental exploratoria y el fenómeno 
de flotación ayudaron a que un estudiante con diversidad funcional fortaleciera sus habilidades de pensamiento científico.

En lo que sigue, presentaremos los resultados del trabajo realizado por una maestra en torno a Carlos, un niño con diagnóstico de trastorno de déficit de atención, hiperactividad e impulsividad (TDAHI) y trastorno mixto de habilidades escolares (TMHE).

\section{Descripción de la experiencia}

\section{Desde un estudio de caso}

Esta propuesta se desarrolla mediante un estudio de caso único que permita comprender la promoción de habilidades de pensamiento científico del estudiante, teniendo en cuenta las características de este estudio particular, que se realiza a profundidad y desde la complejidad que surge de la singularidad en el seguimiento de un estudiante con características específicas, estableciendo un puente entre la teoría y la práctica (Stake, 2007).

También conviene subrayar que aunque los resultados de esta intervención no se pueden extrapolar, debido a la individualidad de los casos de niños con diversidad funcional, definitivamente los resultados de esta propuesta pueden brindar información importante que permita analizar, interpretar y direccionar procesos de intervención en casos con problemáticas similares.

\section{Diseño de investigación-acción participativa}

El proceso de Carlos se fundamentó en el enfoque metodológico investigación-acción participación (IAP), el cual se eligió por la simultaneidad entre sus componentes. Esto permitió hacer un seguimiento de los avances y necesidades de Carlos, tomar decisiones para resolver problemas, adaptar las prácticas experimentales e interpretar lo "que sucede" desde el punto de vista de los participantes, en este caso la maestra y Carlos. Otro aspecto importante del diseño metodológico usado para esta propuesta es que recurre a los métodos y las técnicas tradicionales de investigación social; sin embargo, lo hace de una manera flexible, con ciertas peculiaridades que resultan del hecho de involucrar a los participantes y los fines que persigue.

\section{El caso de Carlos, niño con diversidad funcional}

Inicialmente las condiciones de los diagnósticos fueron relevantes para este estudio de caso, dado que Carlos requiere mayor apoyo para desarrollar actividades que son propias de su edad, pero que representan dificultad para él, como lectoescritura, manejo de instrumentos y realización operaciones matemáticas. Otros aspectos en los que también necesita apoyo son su convivencia social, 
seguir instrucciones, atender a clase y autocontrolar su hiperactividad e impulsividad. Esta comprensión de la realidad de Carlos provocó que la maestra planteará una propuesta que le permitiera enseñar ciencias en medio de la diversidad de aprendices y al mismo tiempo reconocer su individualidad.

La propuesta se realizó a través de la actividad experimental en un escenario (no aislado) que se adaptó a las características del contexto de Carlos y fue haciendo visible sus habilidades de pensamiento científico como en un espiral ascendente. La experiencia vivida por Carlos y su maestra se narra en cuatro momentos. En el primero, la maestra asocia las características del trastorno de déficit de atención, hiperactividad e impulsividad y el trastorno mixto de habilidades escolares al comportamiento y el aprendizaje de Carlos; en el segundo, la maestra lee los contextos de Carlos en la clase de ciencias; en el tercero, ella detalla la interacción de Carlos con sus compañeros a partir de la actividad experimental y, en el cuarto momento, se evidencian los progresos de Carlos a través de experiencias exploratorias más complejas.

Primer momento: las características del trastorno de déficit de atención, hiperactividad e impulsividad y el trastorno mixto de habilidades escolares asociadas a Carlos

En este punto, la maestra se documentó para comprender las características del diagnóstico de Carlos. Halló muchas relaciones entre las observaciones en clase y la literatura sobre trastorno de déficit de atención, hiperactividad e impulsividad, específicamente en el desarrollo de funciones neuropsicológicas como la memoria de trabajo verbal y no verbal, la autorregulación del afecto, la motivación y activación en las actividades escolares, la inhibición de la conducta que, junto con el control motor, hacen evidentes una intensa actividad motora. En el caso del trastorno mixto de habilidades escolares, el individuo diagnosticado con este trastorno presenta serias dificultades en la lectura, la escritura y habilidades del cálculo aritmético (Oriales, 2000). Todo lo anterior lleva a Carlos mantener un sentimiento interno de nerviosismo e inquietud, tal como lo describe la maestra en su bitácora a propósito de la primera interacción:

Me encuentro con un estudiante tímido, que se expresa con monosílabos, se cubre la cabeza y parte de la cara con su abrigo. Responde a la cualquier interacción con sus compañeros agresivamente por esto es rechazado por sus compañeros, constantemente se pone de pie e interrumpe la clase y difícilmente termina los trabajos que se proponen.

Segundo momento: los diferentes contextos de Carlos

La maestra observó en Carlos aspectos que se resaltan como sus respuestas agresivas, su atención dispersa, poco interés en la clase de ciencias, aislamiento, ensimismamiento y rechazo. En el contexto académico, presentaba bajo rendimiento en todas las áreas de su nivel, dificultades para finalizar tareas escritas y actividades que involucraran el uso de la memoria y de cálculos matemáticos. En su contexto social, se encontró a un estudiante sentado en la última silla del salón, temeroso, callado, solitario, inseguro, apático y rechazado por sus compañeros. En el contexto familiar, se hicieron evidentes problemáticas disfuncionales que también incidían en los resultados académicos.

Dadas las condiciones individuales del estudiante, se decidió enfocar la intervención en sus habilidades, gustos e intereses. Con este punto de partida se proyectó un camino hacia 
la promoción de sus habilidades de pensamiento científico, pero la actividad experimental se guardaba sus sorpresas.

Tercer momento: interacción de Carlos con la actividad experimental exploratoria

La interacción de Carlos con la actividad experimental exploratoria permitió el desarrollo de sus habilidades de pensamiento científico como en un espiral ascendente a través de un proceso que se compila en el tercer y cuarto momento.

Teniendo en cuenta los diferentes contextos de Carlos, la maestra empezó a buscar estrategias que permitieran promover en él y sus compañeros habilidades de pensamiento científico. Así, en un ejercicio de lluvia de ideas durante la clase de ciencias, la maestra identificó que los estudiantes se interesaban por los barcos y los aviones. También desde la primera visita Carlos demostró comodidad en el laboratorio de ciencias y curiosidad por manipular implementos como la balanza de tres brazos, el dinamómetro, las probetas y las pipetas Aprovechando este interés, la maestra motivó a Carlos a interactuar con los implementos del laboratorio, como puede apreciarse en la fotografía 1.

Fotografía 1. Interés del estudiante por los instrumentos del laboratorio

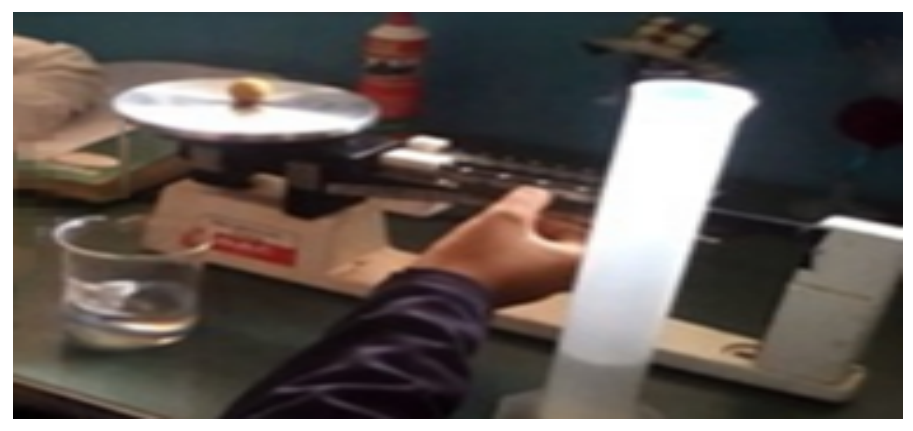

Fuente: grabaciones de clase de Eliana Bolaños.

Inicialmente Carlos manipuló los instrumentos de manera libre manera libre y voluntaria, luego la maestra le ayudó a realizar mediciones básicas y registrar el peso, la masa y el volumen de diferentes objetos; Carlos también logró calcular el volumen de una canica, tal como se registró en las grabaciones de clase:

Maestra: Registra el volumen del agua antes de poner el objeto en la probeta.

Carlos [se acerca a la probeta y lee]: Es 5 mililitros.

Maestra: Ahora introduce el juguete en la probeta y revisa lo que sucedió.

Carlos: Es $7 \mathrm{ml}$.

Maestra: ¿̇Qué crees que sucedió? y ¿̇por qué? 
Carlos: La canica hizo que el agua subiera, porque se suma el volumen de la canica con el del agua inicial.

Maestra: ¿̇Hay alguna forma de saber el volumen de la canica?

Carlos: Si le resto a $7 \mathrm{ml}$ del agua con la canica a los $5 \mathrm{ml}$ del agua sola. Entonces el volumen es $2 \mathrm{ml}$.

Rápidamente aprendió a leer los datos de la balanza sumando las medidas de los tres brazos, aun con cifras decimales. Al final adquirió tal destreza y comprensión del funcionamiento de estos implementos que lograba calibrarlos y repararlos.

Con el propósito de captar el interés de Carlos por los barcos y submarinos, la maestra adaptó a la clase una propuesta de García et al. (2005), quien plantea algunas estrategias para realizar actividades experimentales sobre el fenómeno de flotación de los cuerpos. Para ello, inicialmente asocia el concepto de equilibrio al fenómeno de flotación y plantea la siguiente pregunta: ¿̇Dónde es más seguro navegar: en un barco grande o en un barco pequeño? Luego propone una actividad donde los estudiantes predicen y argumentan si un cuerpo flota o se hunde. Al finalizar, se pudo apreciar en las respuestas que los estudiantes atribuían al peso del objeto su capacidad para flotar o hundirse.

La fotografía 2 registra los barcos de papel de diferente tamaño y el uso que le dieron a las canicas, ubicándolas en los dos barcos para ver cuál resistía más antes de hundirse. En los registros fílmicos, se observa que Carlos trabajaba solo; sin embargo, en el momento de emplear la balanza, se presentó un punto de inflexión para esta investigación. Los estudiantes tuvieron inconvenientes porque desconocían el uso de este implemento. Ante esta situación Carlos, quien se había convertido en un experto debido a su curiosidad, interés y habilidades operativas para obtener datos con precisión en sus medidas, construir montajes y calibrar balanzas, de manera voluntaria se precipitó a ayudar a todos los compañeros, explicándoles cómo hacer las mediciones de manera correcta. Aprovechando esta situación, la maestra lo nombró monitor de la actividad. Cabe aclarar que desde este día Carlos nunca volvió a trabajar solo en clase de ciencias, pues sus compañeros querían hacer equipo con él.

Fotografía 2. Comparando la superficie de sustentación de un barco.

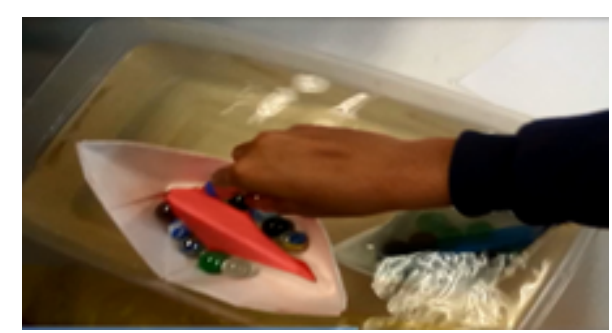

Fuente: grabaciones de clase de Eliana Bolaños y Edwin García.

La destreza de Carlos con los implementos del laboratorio trajo para él otros beneficios, entre ellos la autoconfianza necesaria para que, en medio de la socialización de la experiencia donde todos los estudiantes expresaban lo que vivieron sobre el fenómeno, Carlos levantara su mano para argumentar: "El barco más grande flota mejor, porque resiste más peso". Con esta respuesta claramente hacía referencia a la superficie de sustentación del barco. A partir de ese momento los avances de Carlos en las actividades experimentales se hicieron evidentes. 
Cuarto momento: el progreso de Carlos

Para promover habilidades de pensamiento científico aún más complejas en Carlos y sus compañeros, se propusieron las actividades experimentales en las que García (2005) asocia el volumen, la forma y el material de los objetos con el empuje o resistencia del agua. Para ello, se inició con actividades en las que los estudiantes hallaron la variación de la lectura del peso de un objeto en un dinamómetro dentro y fuera del agua. Durante la socialización de la experiencia, como consta en las grabaciones de clase, Carlos expresó:

Maestra [pregunta dirigiéndose a todo el grupo]: ¿̇por qué es diferente el peso del objeto cuando se sumerge en el agua?

Carlos [levantó su mano y argumentó]: El agua parece tener una fuerza que empuja los objetos hacia arriba.

Maestra: ¿̇Quieres explicarnos un poco más esa afirmación?

Carlos: Sí, vea por ejemplo en la piscina yo puedo cargar a mi abuelo, por eso yo digo que el agua ayuda a que los objetos floten.

Fue así como poco a poco, Carlos fue evidenciando su habilidad para argumentar en público sobre los sucesos observados en la experiencia.

En las actividades adaptadas según la estrategia de García et al. (2005), se asocia el material con el que se construyen los barcos. Para ello, se emplearon dos objetos con igual peso, forma y volumen, pero diferente material: uno es de aluminio y el otro de hierro. Se solicitó a los estudiantes que compararan con el dinamómetro su peso en el agua. Durante la socialización de la actividad se generó el siguiente diálogo:

Maestra: Después de realizar la comparación de los dos objetos en el dinamómetro fuera y dentro del agua, ¿̇con cuál material eligen construir un barco?

Carlos: Yo lo construiría de acero, porque la pesita de acero flota mejor que la de aluminio.

Maestra: ¿̇Por qué eliges este material?

Carlos: Porque cuando ponemos el dinamómetro en el agua el acero pesó 6 gramos y el aluminio 9,2 gramos aproximadamente.

Maestra: ¿̇Por qué crees que los datos del acero son menores que los del aluminio en el dinamómetro cuando lo sumerges en el agua?

Carlos: Yo creo que, como las pesitas tienen el mismo peso y forma, entonces puede ser que el agua le ayuda más a la de acero a flotar, por eso los barcos los construyen de acero, el acero es más fuerte y flota mejor. (Grabaciones de clase de Eliana Bolaños) 
Con esta afirmación Carlos evidencia que puede dejar de considerar las variables que se vienen trabajando y pensar en las características del material de los objetos, lo cual lo lleva a controlar las diferentes variables que hacen que un objeto flote o se hunda. De igual manera, otra habilidad que Carlos mostró en público fue formular hipótesis.

En una ocasión, los estudiantes exploraron los cambios en las condiciones del medio de acuerdo a la cuarta propuesta de García et al. (2005). A continuación se describe la hipótesis que planteó Carlos sobre el fenómeno:

Maestra [solicita a los estudiantes que se organicen en equipos y planteen una hipótesis sobre cómo hacer flotar un huevo en un recipiente con agua]: Antes de que empiecen a experimentar, por favor, el orador del equipo nos cuenta su hipótesis para escribirla en el tablero y comprobarla con los resultados.

Carlos [como orador de su equipo planteó la siguiente hipótesis]: Yo creo que el huevo va a flotar si le mezclamos sal en el agua... porque ya no será la misma agua que te- níamos al inicio, será agua con sal, por eso los barcos grandes y pesados flotan en el mar, porque el agua salada es más densa.

(Grabaciones de clase de Eliana Bolaños)

Esta escena ilustra que Carlos había pasado de ser un estudiante rechazado, sin interés en la clase y callado, a ser un estudiante que manipulaba instrumentos, preguntaba, reflexionaba, planteaba hipótesis y argumentaba sobre el fenómeno de flotación. Sin embargo, el desarrollo de todas estas habilidades evidenció que era necesario dotar aún más de significado las actividades para avanzar en el grado de complejidad de estas habilidades en Carlos y todos sus compañeros. Para esto, se planteó un reto en el que se invitó a los estudiantes a diseñar y construir un artefacto que flotara.

Carlos se tomó en serio el reto y diseñó, construyó con material reciclado, experimentó, probó, desbarató, volvió a diseñar, resolvió problemas, para nuevamente armar un maravilloso submarino que emergía a la superficie cuando les inyectaba aire a unos globos desde afuera con una manguera (véase figura 1).

Figura 1. Diseño final de submarino

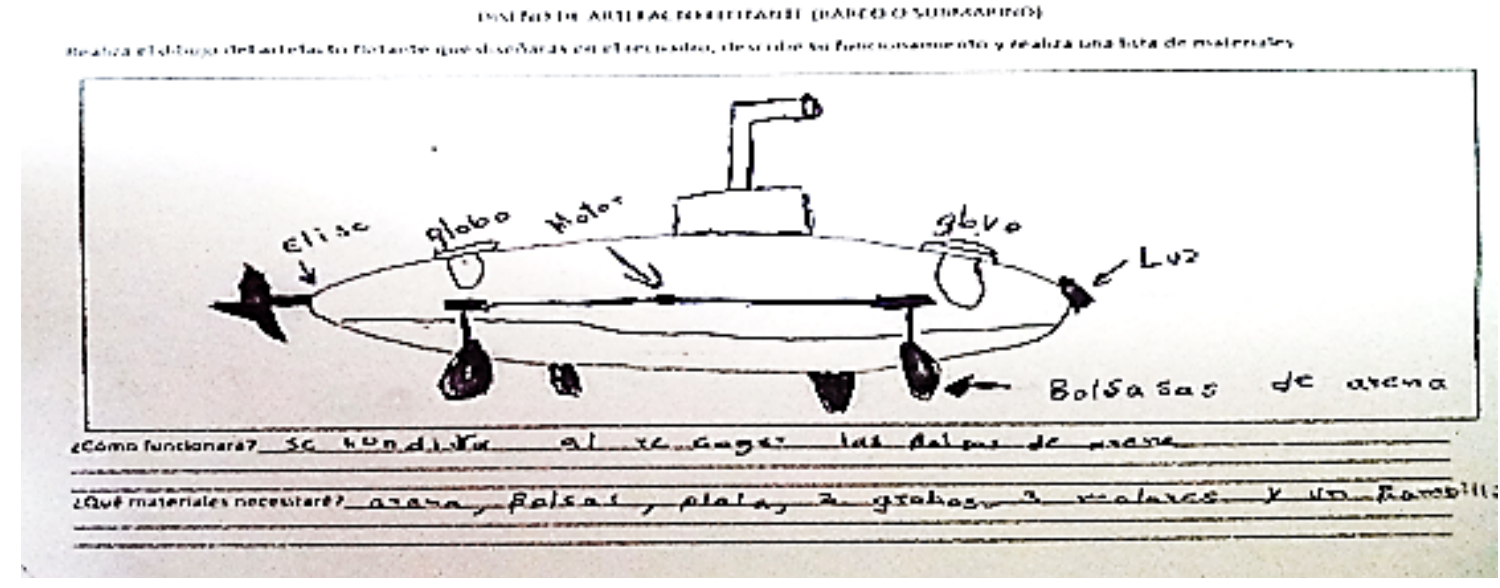

Fuente: diseño elaborado por Carlos Fotografía de Eliana Bolaños y Edwin García 
El día de la sustentación los estudiantes explicaron lo siguiente:

Maestra: Descríbenos tu diseño, los materiales que empleaste y su funcionamiento.

Carlos: Mi diseño es un submarino, que puede hundirse y flotar; los materiales que usé fueron un envase de gaseosa, bolsas con arena, una manguera, cinta, dos globos. Lo que yo creo es que cuando ponga el submarino en el agua, por la forma y el peso del agua y la arena que hay en la botella, se va a sumergir. Luego para que suba a la superficie yo voy a inflar los globos y como el aire es menos denso que el agua, el submarino sale a la superficie.

Maestra: El submarino se ve un poco diferente al dibujo. ¿̇Qué variaciones le hiciste?

Carlos: Es que lo probé antes en mi casa y no se hundía, entonces tuve que resolver varios problemas; para asegurarme de que se hundiera le puse la arena y agua por dentro. Luego para ayudarle a la fuerza del agua en el momento de flotar le puse madera, porque la madera siempre flota y unas pesas de acero que flotan mejor, pero también le dan estabilidad para que no se voltee.

Carlos no solo demostró que el submarino funcionó tal como lo había predicho, sino también su creatividad y habilidades de pensamiento científico, como resultado de las actividades experimentales exploratorias. Finalmente, Carlos socializó este diseño en la feria de la ciencia con algunos compañeros; ellos explicaron el fenómeno de la flotabilidad de los cuerpos a toda la comunidad educativa.

\section{Sistematización y análisis de la experiencia}

\section{Las narrativas experimentales cuentan una historia en el aula de clase diversa}

Para capturar los detalles de cada intervención se hicieron registros fílmicos y escritos en la bitácora del maestro y del estudiante. Con toda la información recogida se construyeron narrativas experimentales, que fueron útiles al hacer un rastreo que inicialmente se centró en las habilidades de pensamiento científico (HPC) de Carlos, pero durante el proceso se evidenciaron otras, como las habilidades socioafectivas y la autorregulación de su comportamiento durante las actividades experimentales. De esta manera, se capturaron momentos definitivos que marcaron la intervención. Por ejemplo, al finalizar las narrativas experimentales la maestra cuenta: "Es grato ver cómo Carlos se expresa en público y responde preguntas de las personas que se acercan a su mesa de exposición durante la feria de las ciencias".

Las narrativas experimentales también se convirtieron en un insumo para definir las conclusiones de la investigación a través del siguiente proceso de análisis. Primero se construyeron redes sistémicas al inicio, durante y al final de 
la intervención, que recogen la información relacionada con las categorías Habilidades de pensamiento científico (HPC), Actividad experimental (AE) y Estudiantes con diversidad funcional (véase figura 2).

\section{Figura 2. Ejemplo de red sistémica de habilidades de pensamiento científico}

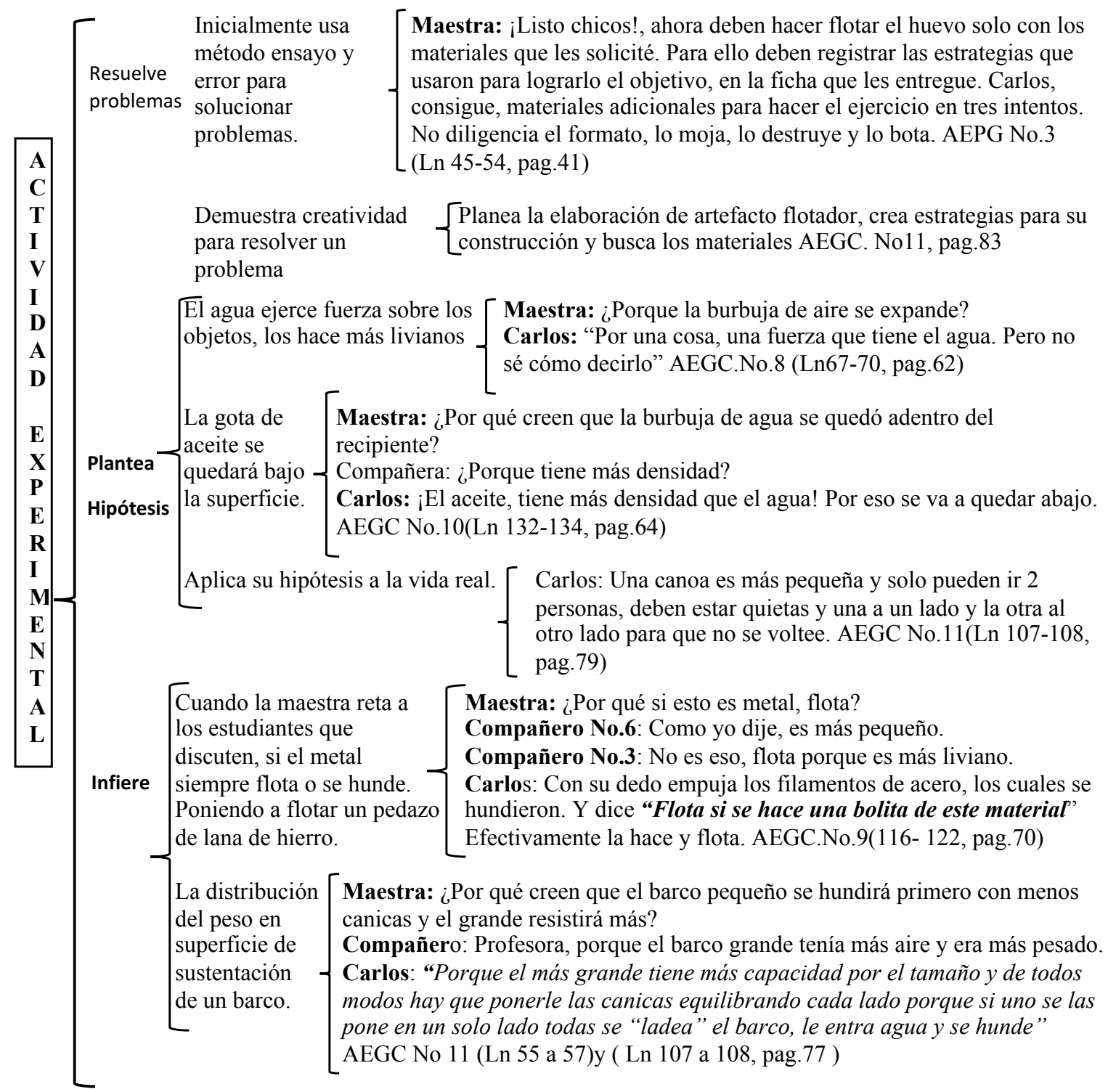

Fuente: elaboración propia a partir de las narrativas experimentales.

AEPG: actividad experimental pequeños grupos

AEGC: actividad experimental grupo completo

Demuestra creatividad
para resolver un $\left\{\begin{array}{l}\text { Planea la elaboración de artefacto flotador, crea estrategias para su } \\ \text { construcción y busca los materiales AEGC. No11, pag.83 }\end{array}\right.$

El agua ejerce fuerza sobre los [ Maestra: ¿Porque la burbuja de aire se expande?
Carlos: "Por una cosa, una fuerza que tiene el agua. Pero no sé cómo decirlo" AEGC.No.8 (Ln67-70, pag.62)

La separación por grupos de las actividades se debe a que en el salón de clase asistían en el momento de la actividad 52 estudiantes

Ln: línea que remite la ubicación de la frase en la narrativa experimental 
Luego, a partir de las redes sistémicas de la figura 2, se establecieron teorías referentes a las debilidades, oportunidades, fortalezas y amenazas, que se observaron durante este proceso, para diligenciar una matriz DOFA ${ }^{1}$ en cada categoría: Habilidades de pensamiento científico (HPC), Actividad experimental (AE) y Estudiantes con diversidad funcional. Finalmente este proceso de análisis permitió establecer las siguientes conclusiones.

\section{Consideraciones finales}

\section{La historia de Carlos no finaliza aquí, pero podemos concluir que...}

La maestra investigadora registró el desarrollo de habilidades de pensamiento científico de un estudiante con diversidad funcional a través de la actividad experimental, en la que se relacionaron aspectos del entorno académico, social, sus gustos e intereses y las características de su diagnóstico al estudio del fenómeno de flotación. Desde su perspectiva, es importante resaltar que aunque este proceso se llevó a cabo en medio de la diversidad de estudiantes en el aula, con el fin de obtener información relevante de la presente investigación, el estudio de caso de un niño permitió visibilizar las siguientes oportunidades y fortalezas de las actividades experimentales exploratorias que puede ser utilizadas en el aula diversa.

La actividad experimental exploratoria ofrece a los estudiantes con trastorno de déficit de atención, hiperactividad e impulsividad y el trastorno mixto de habilidades escolares la oportunidad de desarrollar progresivamente habilidades de pensamiento científico. Esta intervención se inició con la estimulación de las habilidades básicas como manipular instrumentos del laboratorio, inferir, argumentar y establecer hipótesis, las cuales se catapultaron en un espiral ascendente de habilidades que se describe en la figura 3. Estas habilidades son cada vez más complejas y llevaron al estudiante a hacer uso de su creatividad e ingenio para pensar, diseñar, resolver problemas, experimentar y construir un artefacto en relación con el fenómeno estudiado. Igualmente la actividad experimental le permitió hacer diferentes cálculos matemáticos al determinar el volumen de un cuerpo, usar cifras decimales en mediciones de alta precisión con una balanza de tres brazos y comparar datos del peso de un objeto con un dinamómetro fuera y dentro del agua. Al mismo tiempo, le ayudó a desarrollar su comunicación oral al expresar sus ideas, hipótesis y argumentos, producto de la interacción con el fenómeno. 
Figura 3. Espiral ascendente de habilidades de pensamiento científico

\section{Figura 3. Espiral ascendente de habilidades de pensamiento cientifico}

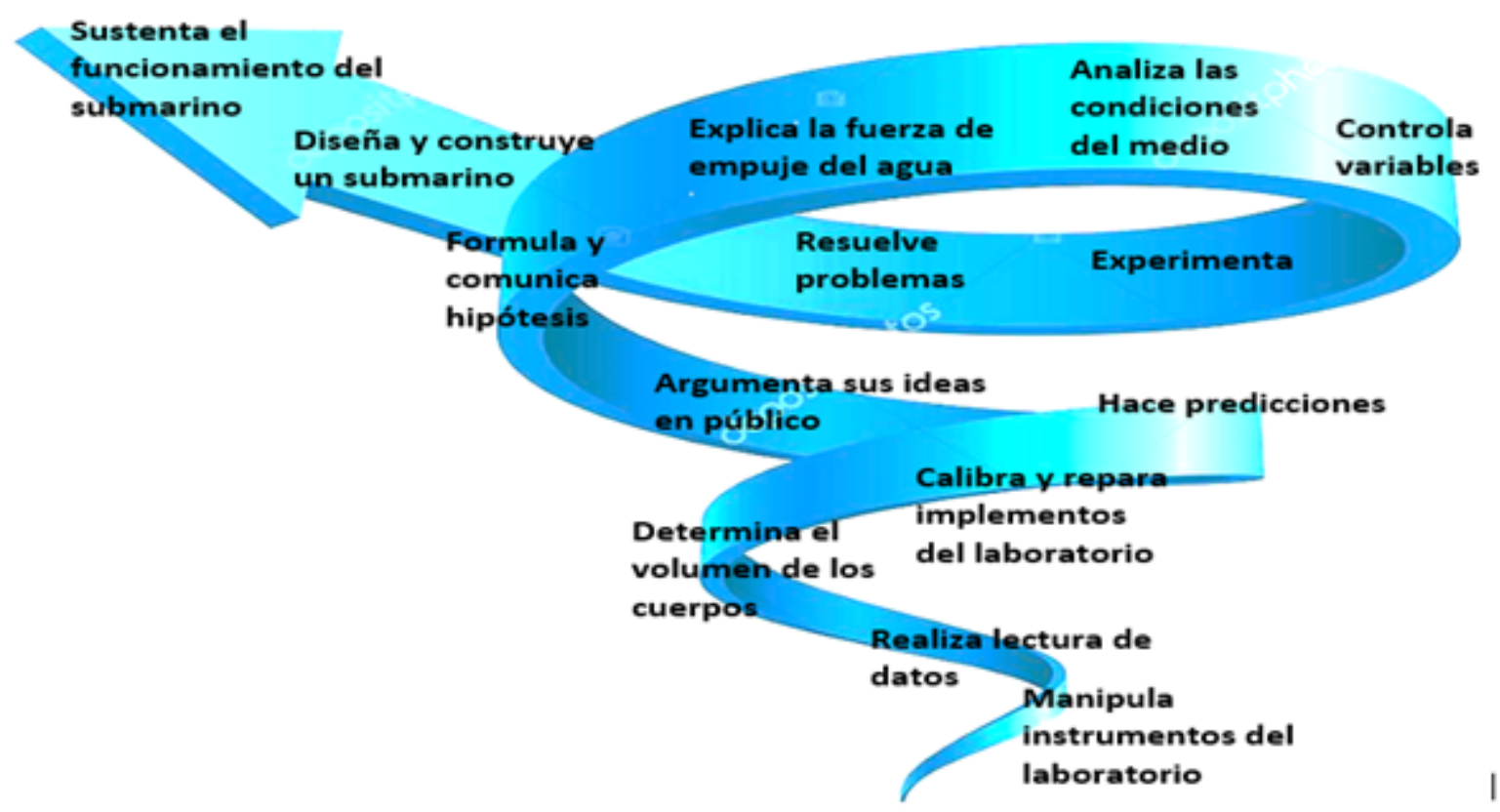

Fuente: elaboración propia.

Asociar el concepto de flotación de los cuerpos a la intervención brindó a los estudiantes la oportunidad de vivenciar un fenómeno cotidiano fácilmente recreado en el aula de clase, lo cual ayudó a promover las habilidades de predecir y comprobar sus afirmaciones, explorar nuevos interrogantes y experimentar para responderlos. Al mismo tiempo, fue posible flexibilizar el concepto debido a que la actividad experimental permitió comprender la esencia del fenómeno, sin recurrir a medios escritos, cálculos y explicaciones magistrales, en las que estudiantes con el trastorno de déficit de atención, hiperactividad e impulsividad presentarían dificultad.

El seguimiento del estudiante evidenció que la actividad experimental exploratoria fortalece la autorregulación de la conducta. Primero, al relacionar sus gustos en situaciones cotidianas sobre la flotación se logró captar su interés y tornar personal la experiencia al vivenciar los sucesos propuestos en torno al fenómeno de flotación. Segundo, se ampliaron los espacios de atención del estudiante durante las actividades, gracias a la reflexión continua sobre sus actitudes y comportamientos, mediante el análisis de las filmaciones y las estrategias de autorregulación (rutinas, acuerdos de clase, pausas activas, compañeros polo a tierra, señales para autocontrol), lo que hizo consciente al estudiante de su capacidad para autorregular su hiperactividad e impulsividad en medio de la clase.

Sorprendentemente, gracias a la actividad experimental, también se evidenciaron avances de las habilidades socioafectivas del estudiante, quien pasó de la timidez, la tristeza y el aislamiento a estar motivado, tener mejor autoestima, así como a sentirse valorado y reconocido por sus compañeros, maestros y familiares. Esto se logró gracias a la cercanía 
y adaptabilidad de la actividad experimental exploratoria y a las actitudes constructivas de la relación empática y adecuada entre la maestra y el estudiante, al validar sus esfuerzos, comprender sus dificultades y reforzar cada pequeño avance.

Es importante resaltar el papel de la afectividad, vista como una relación adecuada entre maestro y estudiante. Esto permitió reconocer la otredad y valorar desde su individualidad a cada uno de los integrantes del aula desde sus diferentes habilidades. Para efectos de este estudio de caso, se destaca la habilidad afectiva que conecta a los estudiantes y sus diversas capacidades a través de la actividad experimental exploratoria. Esto es evidente en este estudio de caso en el que se describe a una maestra interesada en brindar oportunidades para promover en Carlos y sus compañeros habilidades de pensamiento científico.

Un último aspecto se refiere al trabajo en equipo, el cual beneficia las habilidades socioafectivas de estudiantes con diversidad funcional y de sus compañeros, al aprender colaborativamente, compartir observaciones, elaborar hipótesis, discutir métodos y resultados. Desde lo individual, el estudiante con diversidad funcional mejora sus habilidades de pensamiento científico, habilidades sociales, autoestima y motivación; desde lo grupal, con los compañeros de clase, fortalece la tolerancia y el respeto por la diferencia. Lo anterior podría explicarse porque durante el proceso se fue gestando un cambio en la atmósfera del aula, se fueron erradicando las acciones segregadoras y abriendo espacio al reconocimiento por las formas diferentes de aprender.

Finalmente, la maestra adquirió la habilidad de innovar en el aula a través de la continua reflexión sobre su práctica, la cual le permitió minimizar las debilidades y amenazas propias de esta intervención y explorar nuevos caminos para orientar prácticas más equitativas en beneficio de sus estudiantes. Aunque este artículo finaliza con este párrafo, la historia de la maestra y de estudiantes como Carlos se escribe a diario en el aula. Por este motivo solo resta que el presente artículo sea una invitación para ampliar los espacios de investigación sobre la diversidad funcional y avanzar así hacia nuevos retos que ciertamente traerá la enseñanza de las ciencias en el aula de clase diversa.

\section{Referencias}

García, E., Zambrano, A., Solarte, M., Viáfara, R. y Espinosa, A. (2005). Construcción de conocimiento en torno a las ciencias naturales. Universidad del Valle.

García, E. y Estany, A. (2010). Filosofía de las prácticas experimentales y enseñanza de las ciencias. Revista Praxis Filosófica, (31), 7-24.

García, E. (201 1). Retórica y enseñanza de la física. Lenguaje, conocimiento y comunicación [conferencia]. IV Congreso Internacional de Enseñanza de la Física. Bogotá. 
García, E. (2012). Modelos de explicación, basados en prácticas experimentales: aportes de la filosofía historicista. Revista Científica, (14), 89-96.

García, E. G. (2015). Línea de investigación en estudios socioculturales y enseñanza de las ciencias. En A. Claret (comp.), Estatuto epistemológico de la investigación. Universidad del Valle.

García, E. G. (2018). La actividad experimental en la enseñanza de las ciencias. El caso del electromagnetismo. Universidad del Valle.

Harlem, W. (1998). Oportunidades de aprendizaje para todos los alumnos. Enseñanza y aprendizaje de las ciencias (pp. 113-134). Ediciones Morata.

Kahn, S. (2018). From access to assets: Strength-based visions for inclusive science education. En M. Koomen, S. Kahn, C. Atchis y T. Wild, Towards inclusion of all learners through science teacher education (pp. 105114). Brill Sense.

López, M., Echeita, G. y Martín, H. (2009). Concepciones sobre el proceso de inclusión educativa de alumnos con discapacidad intelectual en la educación secundaria obligatoria. Cultura y Educación, 21 (4), 485-496.

López, M., Echeita, G. y Martín, E. (2012). Dilemas en los procesos de inclusión: explorando instrumentos para una comprensión de las concepciones educativas del profesorado. Revista Latinoamericana de Inclusión Educativa 4(2), 155-176.

Puche, R. (2000). Formación de herramientas científicas en el niño pequeño. Arango Editores.

Mastropieri, M., Scruggs, T. y Magnusen, M. (2006). Activities-oriented science instruction for students with disabilities. Learning Disability Quarterly, 22(4), 240-249.
Marín, M. y García, E. (2013). Pensar y actuar en el aula. La construcción de conocimiento a través de la experimentación: el caso de la combustión. En J. F. Malagón, Construcción de fenomenologías y procesos de formalización: un sentido para la enseñanza de las ciencias (pp. 57-86). Universidad Pedagógica Nacional.

McGinnis, R. y Kahn, S. (2014). Special needs and talents in science learning. Handbook of research on science education (pp. 223245). Routledge.

Moin, L., Magiera, K. y Zigmond, N. (2009). Instructional activities and group work in the US inclusive high school co-taught science class. International Journal of Science and Mathematics Education, 7(4), 677-697.

Orjales, I. (2000). Déficit de atención con hiperactividad: el modelo híbrido de las funciones ejecutivas de Barkley. Revista Complutense de Educación, 11(1), 71-84.

Pickering, A. (1986, enero). Against correspondence: A constructivist view of experiment and the real. En PSA: Proceedings of the Biennial Meeting of the Philosophy of Science Association (pp. 196-206). Philosophy of Science Association.

Romañach, J. y Lobato, M. (2005). Diversidad funcional, nuevo término para la lucha por la dignidad en la diversidad del ser humano. Foro de Vida Independiente, 5, 1-8.

Stake, R. E. (2007). Investigación con estudio de casos. Morata.

\section{Forma de citar este artículo:}

García, E. y Bolaños, E. (2022). Contribuciones de la actividad experimental al pensamiento científico de estudiantes con diversidad funcional: la historia de Carlos y el fenómeno de flotación. Tecné, Episteme y Didaxis: TED, (51). https://doi.org/10.17227/ted.num51-12497 
